\title{
Der Basler Humanisten-Arzt Wilhelm Copp (um 1460-1532)
}

\author{
Von Marie-Louise Portmann, Basel
}

\begin{abstract}
Wilhelm Copp (Copus) war zu Beginn des 16. Jahrhunderts ein angesehener Arzt und Gräzist, der im Kreis der Pariser Humanisten eine wichtige Stelle einnahm. Ich möchte nach Copps eigenen Briefen und Übersetzungen, nach Erwähnungen im Schrifttum seiner Zeitgenossen, sowie nach den Ermittlungen E. Wickersheimers, die er im Bulletin de la Société française d'histoire de la médecine 1913 veröffentlichte und die sich hauptsächlich auf die Commentarii Facultatis Medicinae Parisienis ${ }^{1}{ }^{\text {stützen, zunächst }}$ Copps Leben und Werk skizzieren und im zweiten Teil dieser Arbeit auf seine Beziehungen zur Familie Amerbach eingehen.
\end{abstract}

\section{I}

Copps Studienzeit in Basel, Köln, Heidelberg und Paris

Wilhelm Copp war Basler, wuchs in seiner Vaterstadt auf und genoß hier seine erste Ausbildung, denn als Copp im Jahre 1514 Reuchlin vor dem französischen König verteidigte, berief er sich darauf, daß er Reuchlin vor vierzig Jahren gekannt hatte, als er in Basel Copps damaligen Lehrer, Johannes Heberling aus Gmünd, unterrichtete ${ }^{2}$. Nach diesen Angaben muß Copp, wenn nicht gleichen Alters, so doch wenig älter als Erasmus sein, der von 1478 an zu Deventer Schüler war ${ }^{3}$. Im Winter 1478/79 ließ Copp sich in Basel immatrikulieren, denn in der Matrikel ist ein « Wilhelmus Kopp de Basilea» eingetragen ${ }^{4}$. Er studierte zunächst an der Artistenfakultät, wurde Lizenziat und Magister artium ${ }^{5}$. In Basel, wo der Humanismus aufblühte, hatte Copp Gelegenheit, sich als Philologe auszubilden,

1 Manuskripte in der Bibliothèque de la Faculté de Médecine von Paris.

2 Illustrium virorum epistolae.

${ }^{3}$ J.Huizinga, Erasmus, Basel 1928, S. 13.

4 A.Burckhardt, Geschichte der medizinischen Fakultät zu Basel, Basel 1917, S. 21.

${ }^{5}$ E. Wickersheimer, Les médecins de la nation anglaise ou allemande de l'université de Paris aux $\mathrm{XIV}^{\mathrm{e}}$ et $\mathrm{XV}^{\mathrm{e}}$ siècles, Bulletin de la Société française d'histoire de la médecine, Paris 1913, S. 337. 
denn seit 1462 hatte Basel einen Lehrstuhl für «Poesie». Pantaleon berichtet in seiner Prosopographia über Copps Studienzeit in Basel:

«bonarum artium et linguarum fundamenta feliciter in patria iecit»? Daraus könnte entnommen werden, daß Copp auch das Griechischstudium bereits begann, jedoch im Vorwort zu Pauli Aeginetae Praecepta salubria Copo Basiliensi interprete, Paris 1510, nennt er als seine ersten Griechischlehrer Mithridates und Conradus Celtis. Da über einen längeren Basler Aufenthalt dieser beiden nichts bekannt ist, dürfen wir mit P.S.ALLEN 8 annehmen, daß Copp einige Zeit an andern deutschen Universitäten studierte. Von Mithridates weiß man, daß er seit 1484 in Köln lehrte ${ }^{9}$. Mit Konrad Celtis könnte Copp entweder ebenfalls in Köln zusammengetroffen sein, wo jener seit 1477 studierte, oder in Heidelberg, wo Celtis seit 1484 bei Dalberg und Agricola Griechisch- und Hebräischstudien betrieb ${ }^{10}$. Die Vermutung Allens, wonach sich die Beziehungen Copps zu Konrad Celtis erst in Ingolstadt (1492) angeknüpft hätten, erscheint unwahrscheinlich, da er sich nach Wigkensheimers Angaben bereits 1488 nach Paris begab. Er beendigte dort sein Medizinstudium; die Doktorpromotion erfolgte am 17. Mai $1496^{11}$.

Neben seiner ärztlichen Praxis übte Copp eine Lehrtätigkeit als Magister regens aus. Als solcher hatte er an einem der verschiedenen Kollegien Vorlesungen zu halten. Die Commentarii nennen Copp auch im Zusammenhang mit den Streitigkeiten, die die Medizinische Fakultät mit den Chirurgen der Confrérie de Saint-Côme hatte. Diese bildeten in Paris eine Zunft unter dem Patronat des heiligen Kosmas, und durch gute Schulung der Lehrlinge hob sich ihre soziale Stellung. Während nämlich die akademisch gebildeten Ärzte sich hauptsächlich mit innerer Medizin befaßten, waren die handwerklichen Funktionen (Wundarznei, Operationen, Amputationen) Sache der Chirurgen, die als Heilkundige zweiten Ranges galten. Da die

6 R. Wackernagel, Geschichte der Stadt Basel, Basel 1916, Bd. $2^{2}$, S. 596.

7 «er legte die Fundamente der schönen Künste und der Sprachen glücklich in seinem Vaterland », Pantaleon, Pars III, S. 104.

8 Allen I, S. 286.

9 Karl Hartfelder, Unedierte Briefe von Rudolf Agricola, Karlsruhe 1886. Agricola schreibt über Mithridates: «Homo est, ut audio, doctissimus omnium linguarum, Latine, Grece, Hebraice, Caldaice, Arabice, et nescio an omnia numeraverim, peritissimus, preterea teologus, philosophus, poeta (ut in summa dicam) unus in omnibus et omnia in uno. 》

10 Joh. Huemer, in ADB.

11 E. Wickersheimer, a. a. O., S. 337. 
Pariser Chirurgen allmählich eine Konkurrenz zu werden drohten, gebrauchten die Ärzte der Medizinischen Fakultät die Barbiere zur Behandlung ihrer Patienten. Die Barbiere waren «chirurgiens en robe courte», denn sie hatten zur Ausübung ihrer Kunst keine zunftgemäße Schulung erfahren, wie die «chirurgiens en robe longue» von Saint-Côme. Daher richtete die Medizinische Fakultät Kurse für sie ein, in denen die Elemente der Chirurgie vorgetragen und Guy de Chauliac behandelt wurde. Mit der Abhaltung solcher Kurse war Copp beauftragt. Doch da er zum bessern Verständnis seines wenig gebildeten Publikums sich der französischen statt der lateinischen Sprache bediente, fanden die Chirurgen einen Grund, sich bei der Medizinischen Fakultät zu beschweren, und Copp wurde gebeten, seinen Unterricht einzustellen.

Zur Zeit, da Copp nach Paris kam, begann sich dort das Interesse an der antiken Literatur zu regen. Die Renaissancekultur Italiens beeinflußte allmählich auch Frankreich. Neben dem Franzosen Robert GAGUIN ${ }^{12}$ kommentierten die Italiener Fausto Andrelini und Girolamo Balbi lateinische Poeten. Aber erst der Zug Karls VIII. nach Neapel (1494) stellte den richtigen Kontakt mit Italien her und ließ den italienischen Einfluß voll einströmen ${ }^{13}$. In Frankreich griffen die Humanisten vor allem das Griechischstudium auf. Der Grieche Janus Lascaris, den der König aus Florenz, wo er Bibliothekar Lonenzo DE' Medicis gewesen war, nach Paris brachte, erteilte dort Unterricht in der griechischen Sprache. Mit GuILLAUME BUDÉ und LEFÈvRE d'ETAPLES, der bereits in Italien studiert hatte, war Copp unter seinen Schülern ${ }^{14}$, und aus dieser Zeit stammt seine Freundschaft mit diesen beiden Humanisten, für die er auch ärztlicher Berater war.

\section{Copps Beziehungen zu Erasmus und Aleander}

Copp wurde auch bekannt mit Erasmus, der besonders in Paris an Krankheitsanfällen litt. Er spricht von einem «febris quartana», das ihn dort zweimal befiel. In einem Brief vom Januar 1497, den er kurz nach seiner Genesung schrieb, preist er die Hilfe der heiligen Genoveva; durch sie, nicht durch den zugezogenen Arzt, sei er gesund geworden ${ }^{15}$. Im Frühjahr 1500,

12 Ordensgeneral der Trinitarier, Verfasser von De arte metrificandi 1473.

13 J. Plattard, La Renaissance des lettres en France, Paris 1931 (Collection Armand Collin).

14 Nach dem Vorwort zu Pauli Aeginetae Praecepta salubria Copo Basiliensi interprete, Paris 1510.

15 Allen I, S. 164. 
nach der Rückkehr von seinem ersten Aufenthalt in England, wiederholt sich die Krankheit, doch vertraut er diesmal nicht nur auf die heilige Genoveva, sondern auch auf seinen Arzt, Wilhelm Copp, «medicum non modo pertissimum, verumetiam amicum fidum et Musarum, quod rarissimum est, cultorem ${ }^{16}$. Als Erasmus 1506 die Reise nach Italien antrat, widmete er Copp die Ode «De Senectutis incommodis» ${ }^{17}$, die er auf dem Wege verfaßte. Wie sehr Erasmus Copp schätzte, geht auch aus einem Brief hervor, den er viel später, im August 1526 aus Basel an ihn schrieb. Darin beklagt er sich über die Basler Ärzte, die ihm in seiner Krankheit kein wirksames Mittel empfehlen konnten. Erasmus bittet Copp, ihm wenigstens schriftlich beizustehen und bedauert, daß es ihm wegen mangelnder Gesundheit und Kriegsgefahr nicht möglich sei, ihn aufzusuchen ${ }^{18}$.

1503 hatte Lascaris, 1506 Erasmus Paris verlassen. Doch 1508 kam Aleander, der gelehrte Italiener und spätere Nuntius, nach Paris, um Griechischkurse zu geben. Der Entschluß dazu mag im Verkehr mit Erasmus gereift sein, den er in Venedig kennenlernte. Von dort ${ }^{19}$ nahm er die nötigen Bücher mit, denn es fehlte in Paris noch an Lehrmitteln und Editionen von griechischen Texten. Aleander, dessen Vater ebenfalls Arzt war und sich von seinem Sohn die Werke des Hippokrates und Galen hatte erklären lassen, trat mit Wilhelm Copp in nahe Beziehung, der bei ihm seine Griechischkenntnisse vervollkommnete ${ }^{20}$.

16 «einen nicht nur sehr kundigen Arzt, sondern auch einen treuen Freund und, was sehr selten ist, einen Verehrer der Musen », Allen I, S. 285. Es wäre durchaus möglich, daß, wie Allen meint, Copp schon 1497 von Erasmus konsultiert wurde.

17 Desiderii Erasmi opera omnia, Leiden 1703, tome IV, S. 755.

18 Die üblen Erfahrungen des Erasmus mit den Basler Ärzten spiegeln sich auch in den Familiaria Colloquia, die in demselben Jahre in einer Neuausgabe bei Froben erschienen waren. In einem Gespräch, das in dieser Ausgabe neu hinzukam und über das verschiedenartige Sterben des Georgius Ballearius und des Cornelius Montrus handelt, verspottet Erasmus die zahlreichen, sich gegenseitig widersprechenden Ärzte des Georgius. Von Cornelius heißt es dagegen: «Unicum dumtaxat consuluit, sed non minus bonum virum quam bonum medicum». Man könnte annehmen, daß Erasmus dabei an Copp dachte (Familiaria Colloquia, Basel 1526, S. 720).

19 J. Paquier, Jérome Aléandre de sa Naissance à la fin de son séjour à Brindes, Paris 1900, S. 27.

20 J.Paquier, S. 87. - Aleander widmete dem «vorzüglichen Astronomen und Mediziner» die Ausgabe von Ciceros Schrift De Divinatione, die 1510 bei Gourmont erschien. Aus dem Vorwort geht hervor, daß Aleander dank Copps Freigebigkeit und auf seine Aufmunterung hin den Griechischunterricht fortsetzte, J. Paquier, S. 88. 


\section{Copps Bedeutung als Übersetzer}

Während Budé bestrebt war, nach einer neuen Methode, die eine gründliche Kenntnis der alten Sprachen und die Prüfung der schriftlichen Überlieferung eines Werkes forderte, in seinen Annotationes in libros Pandectarum, Paris 1508, die Irrtümer der Glossatoren zu berichtigen ${ }^{21}$, arbeitete Copp an der Übersetzung ärztlicher Schriften aus dem Griechischen. 1510 erschien in Paris bei H.Stephanus die Übersetzung der Praecepta salubria des Paulus Aegineta, 1512 der Praesagia des Hippokrates, 1513 der Schrift Galens De affectorum locorum notitia ${ }^{22}$. Copp schließt sich mit diesen Übersetzungen jenen Ärzten an, welche die arabische Tradition zu durchstoßen trachteten und ihre ärztlichen Kenntnisse unmittelbar aus den griechischen Quellen selbst schöpfen wollten. Daher war eine gründliche philologische Ausbildung für sie die Grundbedingung ihres Schaffens. Der Anstoß dazu ging von Italien aus. 1490 wurde in Venedig bei Phil. Pintius de Caneto die erste lateinische Galen-Ausgabe gedruckt. Das Vorwort des Übersetzers Diomedes Bonardus von Brixen erwähnt die Bestrebungen italienischer Ärzte, die Originaltexte der antiken Heilkundigen textkritisch zu reinigen. Dahin versuchte man durch Vergleichung der Abschriften zu gelangen, und Bonardus versichert, es sei nicht leicht, sie überall aufzustöbern ${ }^{23}$. Weiterhin besorgten vor allem der Italiener LEONIcenus, der in Ferrara lehrte, und der Engländer Thomas Linacre, der in Padua studierte und später Leibarzt Heinrichs VIII. war, die Übersetzung verschiedener Schriften von Hippokrates und Galen. Als dritter

${ }^{21}$ L.Delaruelle, Guillaume Budé, les origines, les débuts, les idées maîtresses, Paris 1907, S. 96.

22 Vgl. dazu das ausführliche Verzeichnis der von Copp übersetzten Werke in E. WICKERSHEIMERs Dictionnaire biographique des médecins en France au moyen âge, Paris 1936, S. 237f. Die Übersetzungen nach P.Aegineta und Hippokrates widmete CopP den Brüdern Germain und Jean de Ganay, die in Paris als Mäzene der Humanisten wirkten, der eine als Bischof von Cahors, der andere als Chancelier de France. Ihnen hatte auch BuDÉ seine Annotationes zugeeignet, Delaruelle, S. 95.

${ }^{23}$ Bonardus schreibt im Vorwort zu Galeni Opera, Venedig 1490, er habe die Bücher Galens, «qui ob transcriptorum ignorantiam adeo corrupti ac mendosi reperiebantur, ut non medico philosophove titulus librorum sed potius poete decantanti digne attribui posset» zu dem Zweck übersetzt, «ut quod ex fonte haberi possit, ex eius rivulo suscipiatur». Dabei sei er folgendermaßen vorgegangen: «ordinando ac corrigendo quam plurima ab ignavis transcriptoribus depravata.» Er fährt dann weiter: «tantum namque dispersa erant Galieni volumina ut opus non leve fuerit in quam plurimis italie gimnasiis ea perquirere.» 
großer Arzt der neuen Richtung galt seinen Zeitgenossen Wilhelm Copp, wie aus einem Brief des Bonifacius Amerbach hervorgeht: «Medicinae studium in barbarorum castris laborans tribus triumviris, Leoniceno, $\mathrm{Li}$ nacro, Copo, cottidie de postliminio asseritur ${ }^{24}$.

Copps Übersetzungen wurden unter seinen Berufskollegen weitherum benützt. Der Arzt Joannes Antonius in Krakau schreibt am 1.April 1527 an Erasmus: «Hippokrates quem Cratander impressit (1526), dii boni, quam indicat fuisse calvam illius Calvi ${ }^{25}$ animam, qui tam bonum medicum verterit tam male; adeo sunt omnia, praeter ea quae Brentii ${ }^{26}$, Copi et Leoniceni, fatua ${ }^{27}$. RABELAIS nahm in seine griechische und lateinische Ausgabe der Aphorismen des Hippokrates (Lugduni, ap. Gryphium 1532) die Übertragungen von Leonicenus, Brentius und Copp ${ }^{28}$ auf; sie bildeten, wie Plattard schreibt, eine Vulgata der Schriften des Hippokrates. Die Autorität der antiken Heilkundigen, Galen und Hippokrates, war noch maßgebend und unbestritten, und die Renaissance schien sie zunächst neu zu befestigen. Nach kurzer Zeit mußte jedoch die philologische Arbeit der Ärzte vor der Erfahrung zurücktreten. 1533, im Jahre nach Copps Tod, kam der Medizinstudent nach Paris, der die Galen-Tradition durchbrechen sollte: Andreas Vesalius ${ }^{29}$.

Außer der Heilkunde widmete sich Copp den mathematischen und astrologischen Wissenschaften, die eng mit jener verknüpft waren, da den astralen Kräften ein überaus großer Einfluß auf den menschlichen Körper

24 "Dem Studium der Medizin, das sich im Lager der Barbaren abmüht, wird durch die Triumvirn Leonicenus, Linacre, Copp, täglich das Recht zur Heimkehr zugesichert», Monumenta Humanistica Lovaniensia, S. 378. - Auch Johannes Adelphi, der Stadtarzt von Schaff hausen, meint dasselbe in einem Brief vom 28. Februar 1520 an Vadian, worin er ihn um das Verzeichnis seiner Bücher bittet, besonders derjenigen, die über die Medizin handeln «quam (medicinam) vides foeda barbarie pollutam et omnino suppressam ni Thomas Lynacer Britannus atque Copus Basileyensis eam resuscitarent suis mellifluis translationibus, quos o utinam aliquando praeceptores habuissem", Vadianische Briefsammlung II, S. 269.

25 Fabius Calvus von Ravenna. Er übertrug in Rom Schriften des Hippokrates, gedruckt 14. August 1525.

26 Andrea Brenta lehrte und übersetzte in Rom, gestorben 1483.

27 «Ihr guten Götter, wie zeigt doch der Hippokrates, den Cratander gedruckt hat, wie kahl die Seele jenes Calvus war (Wortspiel im Lateinischen), der einen so guten Arzt so schlecht übersetzt hat; so sind auch alle Übersetzungen, außer diejenigen von Brenta, Copp und Leonicenus, einfältiges Zeug », Allen VII, S. 29.

28 J.Plattard, La vie de François Rabelais, Paris 1928, S. 91.

29 M.Roтн, Andreas Vesalius Bruxellensis, Berlin 1892, S. 64-73. 
zugeschrieben wurde ${ }^{30}$. Von der ärztlichen Kunst und dem weitverbreiteten Ruhm Copps zeugt Trithemius: «ad ejus aedes non modo Parisienses tanquam ad unicum salutis remedium confugiunt, sed etiam qui huic nostro regno praesunt audita viri fama ocissime mittentes acciunt, ejus opem sentiunt ${ }^{31}$.

Diese Stelle deutet darauf hin, daß Copp von deutschen Fürsten herbeigerufen wurde, was ihn veranlaßte, von Paris zeitweise wegzureisen ${ }^{32}$.

\section{Copps Stellung unter den Königen Ludwig XII. und Franz I.}

Den Basler Arzt nahm Ludwig XII. in seinen persönlichen Dienst. Die Ernennung zum königlichen Leibarzt erfolgte um das Jahr 1512/1513; denn Trithemius erwähnt die neue Würde Copps noch nicht, jedoch kennt er seine beiden ersten Übersetzungen von 1510 und 1512. Im Jahr 1513 war Copp mit Ludwig XII. auf seinem Zug gegen die Engländer, und im Januar 1515 begleitete er mit den andern Hofärzten den königlichen Sarg zu Grabe. Schon seit Beginn der Regierung Franz I. hatte Copp das gleiche Amt inne wie unter dessen Vorgänger, und er verblieb darin zeitlebens ${ }^{33}$. Seine Stellung gewährte Copp direkten Einfluß auf den König und seine nächste Umgebung, den er benützte zugunsten der Humanisten, die mehr und mehr in Gegensatz traten zu den Theologen der Sorbonne. Es zeigte sich dies im Reuchlin-Streit, als er in Paris 1514 sein Echo hervorrief. Die Sorbonne hatte sich in ihrer Mehrheit gegen ReuchuIn gestellt. Zudem waren Legaten der Kölner Theologen nach Paris gekommen, die auch

30 Pantaleon: «eam artem (medicinae) cum Mathematicis disciplinis tanta felicitate exercere coepit ut in omnium admiratione esset.» Das Bildchen, das er der kurzen Biographie Copps beigibt, stellt diesen mit einem astronomischen Meßgerät dar. Außerdem findet sich in Montfaucons Bibliotheca bibliothecarum manuscriptorum nova, tome I, S. 25 b, eine diesbezügliche Angabe: in der Bibliotheca Reginae Sueciae in Vaticana soll sich von GUILLELMUS CoP BASILIENSIS ein Tractatus astrologicus ex variis antiquis desumptus befinden.

${ }^{31}$ «Zu seinem Hause flüchten nicht nur die Pariser wie zum einzigen Heilmittel, sondern auch diejenigen, die unserem Reiche vorstehen, rufen ihn rasch durch Abgesandte herbei und verspüren seine Hilfe.»

32 Eine Stelle in einem Brief des Erasmus an Etienne Poncher, Allen II, S. 454, könnte darauf hinweisen. Er erwähnt Copp, «quem certatim sibi vindicant hinc Gallia, hinc Germania». Doch Erasmus will damit wohl nur sagen, Copp gehöre seiner Herkunft nach zur Germania, seinem jetzigen Dienst nach beim französischen König aber zur Gallia.

${ }^{33}$ E. Wickersheimer, Bulletin de la Sociêté française d'histoire de la médecine 1913, S. 338. Giovio, S. 292. 
Ludwig XII. in ihrem Sinne beeinflussen wollten ${ }^{34}$. Ein Brief vom 25. August 1514 an Reuchlin gibt darüber Aufschluß, wie Copp vor dem König für ihn eintrat. Als ein Bischof den Gegnern das Wort geredet hatte und Copp vom König befragt wurde, ob er Reuchlin kenne, rühmte er seine Gelehrsamkeit und seine Schriften, indem er sich darauf berief, Reuchlin in Basel gesehen und dort von ihm gehört zu haben. Seine Stellungnahme trug ihm den Vorwurf seines Gegners ein, «dixit me pariter judaizare ${ }^{35}$. Weittragenderen Einfluß übte Copp auf Franz I. aus. Mit Budé teilte er die Anstrengungen zur Errichtung des späteren Collège de France ${ }^{36}$, eines Instituts, an dem von eigens berufenen Lehrern regulärer Unterricht in den alten Sprachen erteilt werden sollte. Als Vorbilder wirkten das Institut für junge Griechen Leos X. und das Collegium Trilingue in Löwen ${ }^{37}$. Die Pariser Humanisten ${ }^{38}$ legten dem König nahe, Erasmus nach Paris einzuladen, um hier eine Lehrtätigkeit auszuüben. Copp war es, der von Franz I. den Auftrag erhielt, Erasmus brieflich anzufragen, ob er den Vorschlag annehmen wolle und welche Bedingungen er stelle. Die Antwort des Erasmus war Dank und Lobpreis des Königs, doch mochte der Gedanke, eine Verpflichtung gegenüber dem König einzugehen und in Paris, in der Nähe der Sorbonne, zu lehren, für Erasmus von vornherein wenig Anziehungskraft besitzen; er zog es vor, nicht zu gehen. Erst 1530 nach dem Frieden von Cambrai, der den Konflikt zwischen Franz I. und Karl V. zu einem vorläufigen Abschluß brachte, wurden «lecteurs royaux» eingesetzt. Man darf vermuten, daß Copp die Bemühungen Budés auch jetzt unterstützte.

In der Zeit kurz vor dem Ausbruch des großen theologischen Streites standen die Schüler des Janus Lascaris auf der Höhe ihres Ansehens. Lefèvre d'Etaples, Budé und Copp galten als die Beschützer der «humaniores litterae». Es war dies auch die Zeit, wo Erasmus für eine kulturelle Blüte von der Regierung Franz I. vieles erhoffte. Nur dann, schreibt er an Etienne Poncher, sei Franz I. ein glücklicher Regent, wenn er nicht über

${ }^{34}$ Bulaeus, Bd. VI, S. 65.

35 «Er behauptete, daß ich ebenfalls judaisiere», Illustrium virorum epistolae. - Im Antwortbrief vom Januar 1515 bittet REUCHLIN seinen «treuen Waffenträger» dringend, alles zu tun, um ihm den König geneigt zu erhalten und teilt ihm die Speierer Sentenz und das Interdikt Grimanis mit.

${ }^{36}$ Lefranc, 1893.

371517 von BusLEIDEN gegründet.

38 Etienne Poncher, der Bischof von Paris; François de Rochefort, ehemaliger Erzieher des Königs; Guillaume Petit, sein Beichtvater, BudÉ und Copp. 
möglichst viele, sondern über vorzügliche Männer herrschen wolle, über solche wie Copp und Budaeus ${ }^{39}$.

Copps führende Stellung unter den Pariser Humanisten ersehen wir auch aus zwei Zeugnissen, die beide aus dem Jahre 1517 stammen. Um diese Zeit befand sich der Kardinal Luigi d'Aragona auf seiner Reise durch Deutschland, die Niederlande, Frankreich und Oberitalien, um seinen Verwandten Karl I. zu besuchen. Sein Sekretär, Antonio de Beatis, der Verfasser eines Reiseberichtes, bemerkt von Paris: «in dicta cita sono questi valenthomini: Jacobo Faber, doctissimo in ogni facultà et latino et greco; Gulielmo Burdeus, regio consiliario, el quale anchora che sia legista ha scripto in altra facultà ${ }^{40}$, et Copus, phisico del Re Christianissimo ben docto in l'una et l'altra lengua ${ }^{41}$. Das zweite Zeugnis findet sich in einem Brief Glareans vom 29. August 1517: «Sum bene acceptus a doctissimis viris Lutetiae inter quos est Budaeus, Copus, Faustus, atque Faber Stapulensis » ${ }^{42}$.

Während Lefèvre D'Etaples in den folgenden Jahren im Kampf mit den Lehrern der Sorbonne stand, Budé am französischen Hof in verschiedenen Ämtern eine Rolle spielte und mit neuen Publikationen hervortrat, ist von Copp fast nichts bekannt ${ }^{43}$. Es ist anzunehmen, daß der Arzt, der nie theologische Fragen bearbeitet hatte, nicht in offenen Streit mit der Sorbonne geriet, hatte er doch auch im Reuchlin-Streit für die Persönlichkeit dieses Humanisten, die er hochschätzte, nicht aber für seine Lehrmeinung Partei ergriffen. Doch mußte er verdächtig erscheinen wegen seiner Vertrautheit mit der griechischen Sprache, die oft als Sprache der Häresie bezeichnet wurde ${ }^{44}$. Copp blieb in Verbindung mit seinen Freunden Erasmus, Ludwig Ber und Aleander. Zum letztenmal wird er 1532 in einem Brief Bers an Aleander erwähnt: «Copus noster Parisius recte valet ut

39 Allen II, S. 454.

40 Beatis meint wahrscheinlich Budés De Asse, Paris 1514.

41 «In der erwähnten Stadt sind folgende bedeutende Männer: Jacob Faber, sehr gelehrt in jeder Fakultät, sowohl im Lateinischen als auch im Griechischen, Wilhelm Burdeus, königlicher Ratgeber, welcher, obwohl er ein Jurist ist, auch in der andern Fakultät schriftlich tätig war, und Copus, Arzt des Allerchristlichsten Königs, sehr gelehrt in beiden Sprachen.»

42 «Ich bin in Paris sehr gut aufgenommen worden von gelehrten Männern, unter denen Budaeus, Copus, Faustus und Faber Stapulensis sind», Zwinglis Briefwechsel, Opera, Band VII, S. 59.

43 Bulaeus schreibt in der Historia Universitatis Parisiensis sehr unbestimmt: «et favisse quoque videtur Lutheranis », Bd. VI, S. 938.

44 Lefranc, S. 68. 
senex et plenus dierum, quem scio non mediocriter exhilarandum si nonnihil per literas ei significavero de presenti Magnitudinis Tue statu et quid haud temere vel renitente brevi maximo rei publice christiane bono futurum sentiam ${ }^{45}$. In demselben Jahre starb Copp in Paris ${ }^{46}$.

\section{II}

\section{Copps Beziehungen zur Familie Amerbach}

Copps Verhältnis zu Johannes Amerbach (1430-1513), dem bekannten Basler Drucker, und dessen Familie bleibt im Dunkel bis zum Jahre 1501, da Amerbachs zwei ältere Söhne Bruno (1484-1519) und Basilius (1488 bis 1535) nach Paris zogen, um dort zu studieren. Wir können vermuten, daß Copp schon in Basel mit Johannes Amerbach bekannt wurde, der sich dort während Copps Schulzeit niederließ. Doch es finden sich bis zu dessen Besuch in Basel vom September 1503 keine Zeugnisse eines schriftlichen Verkehrs mit dem Basler Drucker. Nur Johannes Heidelberg, der Geschäftsführer des Nürnberger Druckers Anton Koberger, dessen Obhut die Amerbach-Söhne empfohlen waren, erwähnt Copp in seinen Briefen. Er beruft sich darauf, was er zum Wohl seiner Schützlinge unternehme, geschehe auf Copps Rat und Zustimmung ${ }^{47}$; gegen den Willen des Vaters, der sie dem Magister Ber übergeben wollte, einem Basler, der in Paris Theologie studierte und gleichzeitig in den "Artes liberales» Unterricht erteilte, hatte Heidelberg nämlich Bruno und Basilius dem Magister Loreyo anvertraut. Heidelberg hatte eine tiefgehende Abneigung gegen Ber, die sich in seinen Briefen hemmungslos äußert. Am 24. Juni 1503 berichtet er an Johannes Amerbach, er habe gehört, daß Loreyo in Paris nicht seinesgleichen habe, daß er gut sechzig Schüler unterrichte, Ber dagegen, diese «mala bestia», stehe mit seinen Schülern in keinem guten Verhältnis, ja er wäre froh, wenn er sie nie gesehen hätte. Dies alles habe Copp ihm erzählt ${ }^{48}$. Man gewinnt

45 «Unserem Copp, der ein Greis und bejahrter Mann ist, geht es in Paris gut. Ich weiß, daß es ihn nicht wenig freuen wird, wenn ich ihm in einem Brief etwas über deine jetzige hohe Stellungs berichte und über das, was ich vermute, daß es in kurzem zum Heil der Christenheit geschehen wird.» Brief BERs aus Thann, wo er sich seit seinem Weggang aus Basel zeitweise aufhielt, an den Nuntius Aleander in Regensburg, Friedensburg, S. 482.

46 Am 2.Dezember, Wickersheimer, S. 339.

47 Amerbach-Korrespondenz, Bd. I. S. 125.

48 Ebenda, S. 188. 
den Eindruck, daß Heidelberg diese Nachrichten lediglich mit Copps Namen deckte, um ihnen mehr Nachdruck zu geben. Denn Copp stand zu Ber in freundschaftlichen Beziehungen, und in späteren Briefen trägt Johannes Amerbach seinen Söhnen mehrmals Grüße an Copp und Ber zusammen auf ${ }^{49}$.

Deutlich zu ersehen ist aus Heidelbergs Briefen, daß Copp sich um das physische Wohl der Amerbache kümmerte. Es geschah dies wahrscheinlich auf eine Empfehlung des Vaters hin und durch Heidelbergs Vermittlung. Als im Juli 1502 ein großes Sterben herrschte, schrieb dieser nach Basel, Copp habe ihm befohlen, die Amerbache überhaupt nicht ausgehen zu lassen ${ }^{50}$. Heidelberg trug ihnen die nötigen Nahrungsmittel in ihre Camera und bereitete sie dort $\mathrm{zu}^{51}$. Copp verbot ihnen wohl auch, «böses wasser» zu genießen und riet ihnen an, Wein zu trinken, was Johannes Amerbach, der den Grund nicht einsah, veranlaßte, die entstehende Mehrausgabe zu rügen ${ }^{52}$.

Im September 1503 war Copp kurze Zeit in Basel, vielleicht anläßlich der Reise zu einem Patienten ${ }^{53}$. Dies ermöglichte es Johannes Amerbach, sich mit Copp persönlich über seine Söhne zu besprechen, was ihm um so willkommener sein mußte, als die Briefe des Magisters Loreyo in letzter Zeit keineswegs befriedigend lauteten und die Sprechfertigkeit Brunos und den Studieneifer des Basilius bemängelten ${ }^{54}$. Copp unternahm es, den Vater etwas zu beschwichtigen: In einem Brief vom 3. November äußerte er sich durchaus günstig über die Amerbache und ihre Fortschritte im Studium ${ }^{55}$. Wie herzlich die Beziehungen Amerbachs zu Copp sich gestalteten, zeigt am besten der Brief an seine Söhne, den er ihm bei seiner Rückkehr nach Paris mitgibt:

«doctori Guilhelmo Copp commendavi vos ut filios; qui indubie quod mei amore vobis facere poterit faciet; in quem omnem spem ponite. Quare si quis vos molestet ultra debitum vel si quid vos torqueat, quod nemini dicere audeatis, et omnes necessitudines incumbentes eidem doctori propalate uti mihi qui pater sum. Mitis est, bonus est, aequus est et nullum mihi dubium vobis erit consilio et auxilio. Nolite vereri aliquem hominem

\footnotetext{
49 Ebenda, S. 269, 309.

50 Ebenda, S. 148.

51 Ebenda, S. 139.

52 Ebenda, S. 148.

53 Ebenda, S. 199.

54 Ebenda, S. 172.

55 Ebenda, S. 199.
} 
sed ei totum cor aperite et omnes vestros defectus et, ut spero, vobis providebit in honestis et licitis; ubi autem senserits vo errare more pii patris vos corripiet et iter rectum ostendet $»^{56}$.

Copp trat nun unmittelbar mit den Amerbach-Söhnen in Verbindung, und im Frühling 1504 wirkte er mit, daß die zwei Studenten vom Kollegium Lexoviense ins Kollegium Burgundiae hinüberwechseln konnten. Auf die Klagen in den Briefen seiner Söhne, daß sie von Magister Loreyo vernachlässigt würden ${ }^{57}$, hatte sich Amerbach an Copp gewandt, der darauf den Umzug vom einen Kollegium in das andere zustande bringen half ${ }^{58}$.

Weniger glücklich war Copp in der Ausführung eines zweiten Auftrags, den er in Basel übernommen hatte ${ }^{59}$. Er sollte einige Schriften Augustins abschreiben lassen, von denen sich Manuskripte in Paris befanden und die dem Basler Drucker noch fehlten. Der anderweitig beschäftigte Arzt ließ jedoch irrtümlicherweise nicht das Gewünschte kopieren. Die Abschriften von De innocentia und Sextum musicum, die er Amerbach Ende 1504 zustellte, besaß dieser schon vierfach und mußte nun die unnützen Auslagen bedauern ${ }^{60}$.

Im Sommer 1505 war Copp wahrscheinlich wieder längere Zeit von Paris abwesend, denn als Basilius und Bruno ernsthaft erkrankten ${ }^{61}$, scheint Copp nicht konsultiert worden zu sein. Johannes Amerbach sandte seinen Söhnen selbst Medikamente, Pulver und Salben nach Paris ${ }^{62}$. In seinen Briefen sind die zu bestellenden Grüße allein an Ber gerichtet ${ }^{63}$. Erst im Oktober, als es den Söhnen bereits wieder besser ging, wurde Copp wieder

56 «Dem Doktor Wilhelm Copp habe ich Euch anempfohlen wie eigene Söhne; er wird Euch ohne Zweifel aus Liebe zu mir tun, was er kann; setzt alle Hoffnung auf ihn. Wenn Euch daher etwas lästig ist oder etwas Euch quält, was Ihr niemandem zu sagen wagt, eröffnet alle Eure Bedürfnisse diesem Doktor, wie Ihr sie mir anvertrauen würdet, der ich Euer Vater bin. Er ist milde, gut, gerecht, und er wird, daran zweifle ich nicht, Euch Rat und Hilfe gewähren. Fürchtet nicht irgend einen Menschen, sonder eröffnet Copp Euer ganzes Herz und alle Eure Fehler, und er wird, wie ich hoffe, in allen ehrenwerten und erlaubten Dingen die nötige Vorsorge treffen; wo er jedoch bemerkt, daß Ihr Euch irrt, wird er Euch nach Art eines guten Vaters schelten und Euch den rechten Weg zeigen.» Ebenda, S. 192.

57 Ebenda, S. 206.

58 Ebenda, S. 207, 210.

59 Ebenda, S. 199.

60 Ebenda, S. 232.

${ }^{61}$ Ebenda, S. 257, 258, 260, 262.

62 Ebenda, S. 251, 267.

63 Ebenda, S. 256. 
mitgegrüßt ${ }^{64}$. 1506 kehrten Bruno und Basilius als Magistri artium nach Hause zurück, und aus der erhaltenen Korrespondenz der Amerbache ist nicht darauf zu schließen, daß die Familie in fernerem persönlichen Kontakt zu Wilhelm Copp gestanden hat.

Die zu wenig zahlreichen Quellen erlauben es nicht, mehr über die Beziehungen Copps zu den Amerbachen zu berichten. Doch darf die Stelle in einem Brief des Beatus Rhenanus an Lefèvre d'Etaples vom März 1512 nicht unerwähnt bleiben, wo er die bedeutendsten Humanisten deutscher Städte aufzählt. Als die Basler Humanisten nennt er dort Copp und die Amerbache. Er zählt sie zu jenen Männern «qui reliquis ducum instar viam disciplinarum foeliciter aperiunt ${ }^{65}$.

$$
\text { Quellen }
$$

P.S.Allen, Opus Epistolarum Des. Erasmi Roterodami, Oxford 1906ff. (zit. Allen). Amerbach-Korrespondenz, herausgegeben von A. Hartmann, Basel $1942 \mathrm{ff}$.

Antonio de Beatis, Die Reise des Kardinals Luigi d'Aragona durch Deutschland, die Niederlande, Frankreich und Oberitalien 1517-1518, herausgegeben von L.PASTor, Freiburg 1905.

Desiderius Erasmus Opera Omnia, Leiden 1703

Familiaria Colloquia, Basel 1526.

W.Friedensburg, Beiträge zum Briefwechsel der kath. Gelehrten Deutschlands im Reformationszeitalter, Zeitschrift für Kirchengeschichte XVI, Gotha 1896 (zit. Friedensburg).

Paolo Grovio, Elogia doctorum virorum ab avorum memoria publicatis ingenii monumentis illustrium, Basel 1571.

K. Hartfelder, Unedierte Briefe von Rudolf Agricola, Karlsruhe 1886.

Illustrium virorum epistolae ad J.Reuchlin, Hagenoae 1519.

Monumenta Humanistica Lovaniensia, herausgegeben von Henry de Vocht, Löwen 1934.

Heinrich Pantaleon, Prosopographia heroum atque illustrium virorum totius Germaniae, Basel 1561 (zit. Pantaleon).

Beatus Rhenanus, Briefwechsel, herausgegeben von Ad. Horawitz und K. Hartfelder, Leipzig 1886.

Trithemius, Catalogus scriptorum ecclesiasticorum sive illustrium virorum, cum appendice eorum qui nostro etiam seculo doctissimi claruere, Basel 1531.

Vadianische Briefsammlung, herausgegeben von E.Arbenz und H. Wartmann, Mitteilungen zur Vaterländischen Geschichte, $1890 \mathrm{ff}$.

Zwinglis Briefwechsel, Zwinglii Opera, Bd. VII, herausgegeben von EgLI-FinsLer-KöHLER, Leipzig 1911.

${ }^{64}$ Ebenda, S. 269.

65 «die den übrigen als Führer gedient und ihnen den Weg der Bildung glücklich zugänglich gemacht haben», Briefwechsel des Beatus Rhenanus, S. 41. 


\section{Darstellungen}

Allgemeine Deutsche Biographie, Leipzig 1875-1911 (zit. ADB).

C.E.Bulaeus, Historia Universitatis Parisiensis authore Caesare Egassio Bulaeo, Pari \$ $1665 \mathrm{ff}$. (zit. Bulaeus).

A.Burchardt, Geschichte der medizinischen Fakultät zu Basel, Basel 1917.

L. Delaruelle, Guillaume Budé, les origines, les débuts, les idées maîtresses, Paris 1907 (zit. Delaruelle).

J.Huizinga, Erasmus, übersetzt von W. KaEgi, Basel 1928.

A.Lefranc, Histoire du Collège de France, Paris 1893 (zit. Lefranc).

J.PAquier, Jérôme Aléandre de sa naissance à la fin de son séjour à Brindes, Paris 1900 (zit. Paquier).

J.Plattard, La vie de François Rabelais, Paris 1928.

- La Renaissance des lettres en France, Paris 1931 (Collection Armand Collin).

M. Rотн, Andreas Vesalius Bruxellensis, Berlin 1892.

R. Wackernagel, Geschichte der Stadt Basel, Basel 1907-1924.

E. Wickersheimer, Les médecins de la nation anglaise ou allemande de l'université de Paris aux $\mathrm{XIV}^{\mathrm{e}}$ et $\mathrm{XV}^{\mathrm{e}}$ siècles, Bulletin de la Société française d'histoire de la médecine, Paris 1913.

- Dictionnaire biographique des médecins en France au moyen âge, Paris 1936. 